\title{
Tumour, Torsion or Trauma?
}

\begin{abstract}
M. Nduhiu, MBChB, MBA, Senior House Officer (Surgery), Kenyatta National Hospital and P.G. Mburugu, MBChB, MMed, FRCS(Urol), FEBU, Consultant Urologist, Kenyatta National Hospital

Corresponding author: Dr. M. Nduhiu, P.O. Box 449-00202, Kenyatta National Hospital, Nairobi, Kenya, E-mail: mathengenduhiu@yahoo.co.uk
\end{abstract}

\section{Summary}

The acute scrotum is a common surgical emergency. Laboratory and radiological investigations are key adjuncts to diagnosis but may occasionally mislead. The condition remains a diagnostic challenge for clinicians and fertile ground for litigation. We present a case of a 22 year old who was managed by various levels of clinicians for a 12 day period prior to testicular exploration which finally revealed testicular torsion. Remarkably, the testis was viable and the finding of a hematoma of the epididymis suggested a history of trauma which was not forthcoming from the patient.

The acute scrotum demands expeditious attention and the clinician must aim to reach a definitive diagnosis in the shortest time possible through thorough history taking and physical examination. Scrotal ultrasound with Doppler examination is a useful adjunct, but investigations must not delay emergent scrotal exploration when testicular torsion is suspected clinically. Relevant consultations and referrals must also be urgent where the results of treatment are suboptimal or the evolution of symptoms leads to diagnostic uncertainty.

\section{Introduction}

Testicular torsion was first described by John Hunter in 1810. In an even earlier paper in 1776, he had described a typical case in an 18 year old who later developed torsion of the contra-lateral testis and suffered bilateral testicular atrophy (1). Hunter observed that certain activities increased the risk of torsion in mobile testes, singling out swimming, cycling, parachuting and ice skating. In 1922, Sellheim pointed out that the risk was inherent in sudden rotational movements and in 1923 Tenckhoff added walking and turning during sleep (1).

The most common factor predisposing to testicular torsion is the 'bell clapper' deformity. In this deformity, the tunica vaginalis has a high insertion on the spermatic cord and the testis lies suspended and liable to rotation. Other uncommon associated factors are cryptorchidism, a long mesorchium between the epididymis and the testis (resulting in a horizontal lie of the gonad) and trauma (2). Anderson and Williamson found testicular torsion "clustered around puberty" but noted that it was common in men in their twenties (2). The age distribution has been described as bimodal with one peak in infancy and a much higher peak at age 13 years $(2,3)$. Extra-vaginal testicular torsion predominates in infancy while intra-vaginal torsion is more common in teenage and adulthood (3).

The testis in torsion suffers ischemia and after six hours infarction is observed leading to testicular atrophy if spontaneous de-torsion or active intervention does not occur $(1,3)$. With prolonged periods of ischemia, even when the testis is found viable at operation, and is preserved, subsequent atrophy may still ensue (4). With duration of torsion greater than 12 hours $75 \%$ of the testes were found irreversibly infarcted in the Bristol study (2). The degree of torsion affects the rate of infarction, prolonged preservation being more likely with less than $360^{\circ}$ torsion $(2,4)$.

Sudden onset of severe hemiscrotal pain is the most common first symptom of testicular torsion and may be associated with nausea and vomiting 
(3). The scrotal pain may radiate to the groin, the abdomen, or the thigh, and in about $5 \%$ may only be in the abdomen. In a further $5 \%$ of cases there may be lower urinary tract symptoms especially in the pre-teen age group (2). History of previous episodes of testicular pain and trauma should be sought (5).

Physical examination reveals an elevated testis, usually in horizontal lie on the affected side with scrotal swelling developing in longer duration of torsion. There may be a low grade fever and the cremasteric reflex is usually absent (3). In early presentation, before the onset of scrotal oedema, elevation of the affected testis, anterior location of the epididymis, horizontal lie of the testis and, ipsilateral loss of the cremasteric reflex were found to be the most predictive of torsion (5).

Confirmatory investigations may include a Doppler ultrasound scan to demonstrate decreased perfusion of the testis, and laboratory investigations such as a urinalysis and CRP are useful in ruling out differential diagnoses. Emphasis is however placed on clinical evaluation and there should be no delays in surgical intervention awaiting investigations where torsion is clinically suspected $(5,6)$.

The UK Medical Protection Society has indicated that though not "overly prevalent" misdiagnosis of scrotal swellings, testicular lumps and pain are a regular source of litigation for which experts almost always recommend that the society settle the claim out of court (7). The two most common scrotal conditions leading to litigation were testicular torsion and testicular cancer.

\section{Case report}

A 22 year old college student presented with a 12 day history of left testicular pain and swelling. He reported having woken up to find the left scrotum swollen and painful. This was the first episode and there was no history of coitus in the preceding two weeks. There was no associated nausea or vomiting, no lower urinary tract symptoms, no urethral discharge and no history of trauma. Prior to admission he had received some unspecified treatment at a private clinic on the day of onset, with partial relief of the pain and swelling.

On the third day following the onset of pain he visited our hospital where an ultrasound scan was ordered, which was reported as showing an enlarged hypervascular left testis, with a mass adjacent to it. The radiologist felt the mass was covered by tunica albuginea and had punctate microcalcifications. The radiologist further reported that no features of abscess were noted and the right testis and epididymis were normal (Figure 1), concluding that testicular neoplasm was likely with a differential of testicular torsion. The patient was put on antibitotics and referred to the urology clinic where he was booked to be seen in a week's time upon which visit he was admitted for emergency scrotal exploration.

Figure 1: Ultrasound scan showing the left testis, the mass and the normal right testis

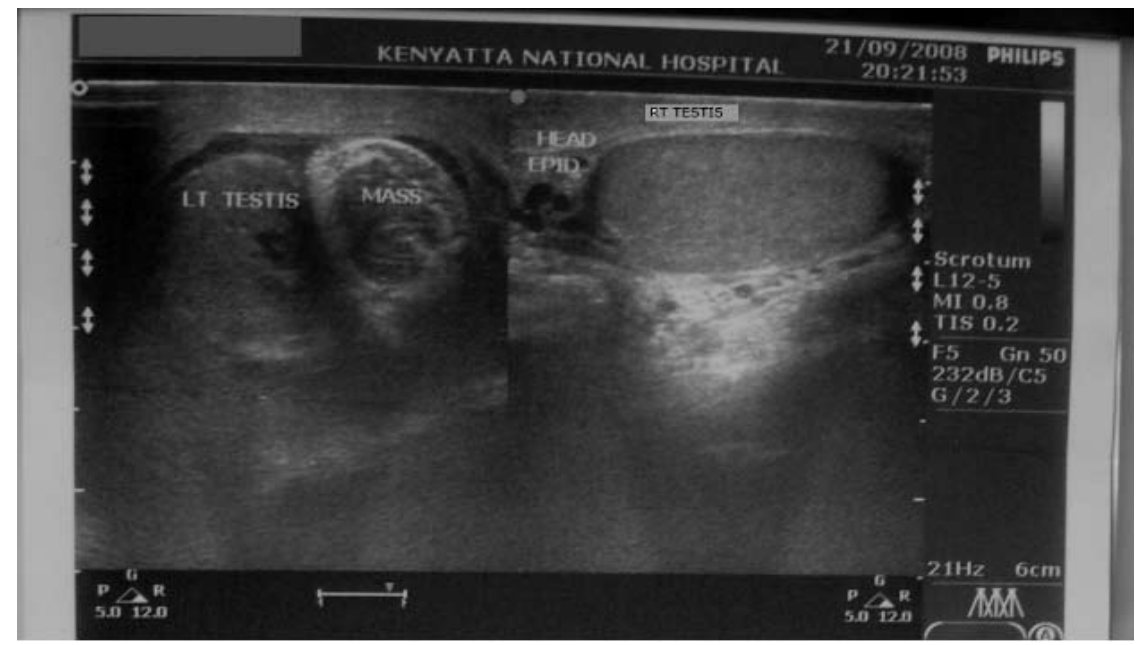


On examination at admission, he was found in fair general condition with normal vital signs; $\mathrm{P} / \mathrm{R}$ 68/min, BP $110 / 70 \mathrm{mmHg}, \mathrm{T} 36.9{ }^{\circ} \mathrm{C}$. Scrotal examination revealed a swollen tender left testis, estimated size $9 \times 6 \mathrm{~cm}$ with a smooth surface and a warmer left hemiscrotum. The right testicle was normal and the rest of the systemic examination was unremarkable. The pre-operative laboratory workup showed a PCV of $47 \%$, human Chorionic Gonadotropin $1.7 \mathrm{mIU} / \mathrm{ml}$ and, Alpha Fetal Protein $4.6 \mathrm{IU} / \mathrm{ml}$ which were all within normal limits. The Lactate Dehydrogenase level was 446 U/L with normal limits of $135-225$ U/L. The urea and electrolytes were within normal.

$\mathrm{He}$ was counseled and gave consent for an inguinal exploration of the left testis, in view of the suspicion of tumour, with possibility of left orchidectomy. He was prepared and taken into theatre on the evening of admission. Under spinal anaesthesia, the left spermatic cord was exposed through a left inguinal skin crease incision and clamped at the deep inguinal ring with a noncrushing intestinal clamp. The left testis was then elevated into the wound and was found oedematous. The tunica vaginalis was incised to find a $180^{\circ}$ clockwise intravaginal testicular torsion with a hematoma at the head of the epididymis (Figure 2). There was no palpable mass within the left testis. The clamp on the cord was thus released and the de-torsed testis quickly showed return of good colour (and upon incision

Figure 2: Showing the testis in torsion and the epididymal heamatoma

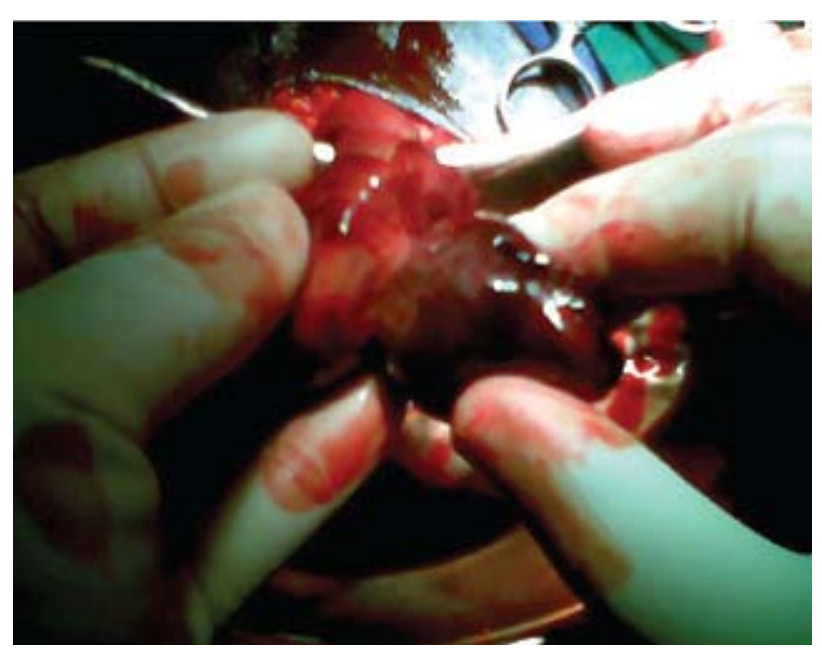

of the tunica albuginea there was immediate brisk arterial bleeding) (Figure 3). The hematoma at the epididymal head was evacuated and the testis repaired, then the inguinal incision was closed in layers.

Figure 3: Showing the testis after detorsion. The colour is restored but the testis appears congested. The epididymis has been turned inferiorly

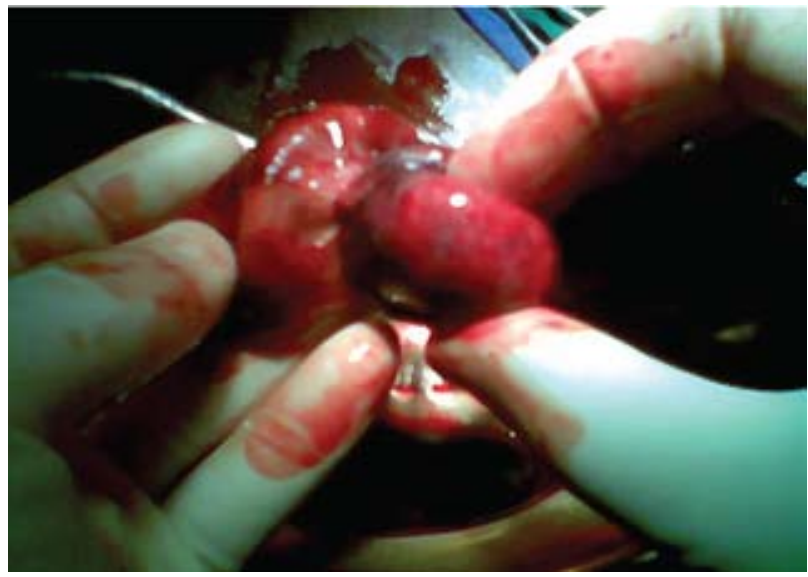

The scrotum was then accessed through a median raphe incision and bilateral orchidopexy performed. The post operative period was uneventful and the patient was discharged on the second post operative day.

\section{Discussion}

This case highlights several important issues in diagnosis and management of the acute scrotum. First, the history in this case was highly suggestive of testicular torsion. It is documented that testicular torsion can occur suddenly during sleep $(1,2)$, and this may occur in up to $5 \%$ of cases (2). The absence of lower urinary tract symptoms should also have pointed to torsion for the first clinician. Did the patient volunteer all the facts preceding the scrotal pain or was there some trauma that occurred in his sleep? Trauma is a long recognized associated factor $(1,2,3)$, supported in this case by the intraoperative finding of a hematoma in the epididymis. Kwong, Nathan and Macdonald have described a very similar case of traumatic testicular torsion in a 15 year old boy injured by a door knob with similar ultrasonographic and intra-operative findings (8). 
Secondly, when did the torsion occur in this case? It has been observed that after six hours of torsion the ischemia is irreversible (3). In a recent prospective study comprising 15 patients, of the six presenting after 10 hours or more, no testis was salvaged and they were uniformly necrotic on histopatholgy (9). It is highly unlikely that a testis can be viable after 12 days in torsion so possibly the torsion either progressed slowly from the time of trauma, as the oedema in the injured epidydimis increased, was intermittent, or the $180^{\circ}$ torsion allowed adequate perfusion.

Thirdly, the ultrasonography report which suggested neoplasm led to a delay in exploration directing us to perform further investigations. Pepe et al found that colour Doppler sonography had a sensitivity and specificity of $86.5 \%$ and $85.3 \%$ respectively versus $100 \%$ and $95.7 \%$ respectively for physical examination in testicular torsion (10). They and other authors conclude that the findings of imaging modalities in the acute scrotum must be viewed in light of the clinical findings $(3,6,10,11)$.

\section{Conclusion}

This case demonstrates that in testicular torsion the clinical evaluation supersedes investigations and scrotal exploration must not be delayed when torsion is suspected and imaging results are equivocal. As in all clinical situations, the clinician must keep an open mind and resist the false sense of finality inherent in reaching a plausible diagnosis. It remains open to debate whether this was a case of traumatic testicular torsion from the onset, or a primary testicular torsion with the resultant testicular enlargement and malposition predisposing the affected testis to secondary trauma.

\section{References}

1. Noske H., Kraus S.W, Altinkilic B.M. et al. Historical milestones regarding torsion of the testicular organs. $J$. Urol. 1998; 159: 13-16.

2. Anderson J.B. and Williamson R.C.N. Testicular torsion in Bristol: a 25-year review. Brit. J. Surg. 1988; 75(10): 988-992.

3. Kapoor S. Testicular torsion: A race against time. Int. J. Clin. Pract. 2008; 62(5): 821-827.

4. Mills S.E., Carter D., Reuter V.E., et al. Sternberg's diagnostic surgical pathology. 2004; $4^{\text {th }}$ Ed. Vol II. Lippincott Williams and Wilkins: Philadelphia.

5. Ciftci A.O., Senocak M.E., Tanyel F.C. et al. Clinical predictors for differential diagnosis of acute scrotum. Eur J Pediatr Surg. 2004; 14: 333-338.

6. Lin E.P., Bhatt S., Rubens D.J., et al. Testicular torsion: twists and turns. Semin Ultrasound CT MRI. 2007; 28: 317-328.

7. Anthony S. Scrotal confusion: focus on diagnosis. Casebook: J. Members Med. Prot. Soc. 2002; 19: 4-10.

8. Kwong Y., Nathan T. and McDonald J. A case of traumatic testicular torsion associated with a ruptured epididymis. Int. J. Urol. 2004; 11: 349-351.

9. Cimador M., DiPace M.R., Castagnetti M., et al. Predictors of testicular viability in testicular torsion. $J$. Pediatr. Urol. 2007; 3: 387-390.

10. Pepe P., Panella P., Pennisi M. and Aragona F. Does colour Doppler sonography improve the clinical assessment of patients with acute scrotum? Eur. J. Radiol. 2006; 60: 120-124.

11. Schalamon J., Ainoedhofer H., Schleef J., et al. Management of acute scrotum in children-the impact of Doppler ultrasound. J. Pediatr. Surg. 2006; 41: 1377 1380. 